\title{
Experimental Tests of Realistic Local Theories via Bell's Theorem
}

\author{
Alain Aspect, Philippe Grangier, and Gérard Roger \\ Institut d'Optique Théorique et Appliquée, Université Paris-Sud, F-91406 Orsay, France \\ (Received 30 March 1981)
}

\begin{abstract}
We have measured the linear polarization correlation of the photons emitted in a radiative atomic cascade of calcium. A high-efficiency source provided an improved statistical accuracy and an ability to perform new tests. Our results, in excellent agreement with the quantum mechanical predictions, strongly violate the generalized Bell's inequalities, and rule out the whole class of realistic local theories. No significant change in results was observed with source-polarizer separations of up to $6.5 \mathrm{~m}$.

PACS numbers: $03.65 . \mathrm{Bz}, 32.50 .+\mathrm{d}, 32.80 . \mathrm{Kf}$
\end{abstract}

Since the development of quantum mechanics, there have been repeated suggestions that its statistical features possibly might be described by an underlying deterministic substructure, a quantum state representing a statistical ensemble of "hidden-variable states." In $1965 \mathrm{Bell}^{1}$ showed that any such "hidden-variable substructure," if local, yields predictions that differ significantly from those of quantum mechanics (QM) in some special situations. Bell's theorem was extended in 1969 by Clauser, Horne, Shimony, and $\mathrm{Holt}^{2}$ to cover actual systems, providing an experimental test for all local hidden-variable theories. Further generalizations ${ }^{3,4}$ have pointed out that determinism is not a crucial feature leading to the conflict with QM. That is, Bell's inequalities have been shown to apply to a broader class of theories: "objective local theories,"3 or "realistic local theories" that can be tested in actual experiments. Such experiments are realizations of Bohm's "Gedankenexperiment," 6 inspired by the famous paper by Einstein, Podolsky, and Rosen. ${ }^{7}$ A source emits pairs of suitably correlated photons (or spin- $-\frac{1}{2}$ particles in a singlet state) that separate. One performs correlated measurements of their polarizations with use of remote polarizers (or Stern-Gerlach magnets) in various orientations. For particular sets of polarizer orientations, the two particle correlations predicted by QM do not obey Bell's theorem.

The first such investigations employed the $2-\gamma$ decays produced by ground-state positronium annihilation. Except for one of them, the experiments $^{8,9}$ agree with the QM predictions. How ever, because of the lack of efficient polarizers for $0.5-\mathrm{MeV}$ photons, strong supplementary assumptions are necessary to interpret these results via Bell's theorem. ${ }^{5}$ Similarly, an experiment ${ }^{10}$ that uses pairs of protons with correlated spins affords results in agreement with $Q M$, but requires similar assumptions.

Pairs of low-energy photons emitted in certain atomic radiative cascades are candidates for better tests. ${ }^{2}$ With a reasonable assumption about the detector efficiencies, ${ }^{2,3}$ the actual experiments constitute a valuable test of local realistic theories via Bell's theorem. So far, four experiments ${ }^{11}$ of this type have been carried out; three of them have agreed with QM predictions. In the most recent such experiment by Fry and Thompson (upholding QM), a high pumping rate of a $(J=1) \rightarrow(J=1) \rightarrow(J=0)$ cascade was attained using a tunable laser, allowing shorter periods of data collection $(80 \mathrm{~min}$ ).

In this Letter we report the results of measurements of the polarization correlations of visible photons emitted in a $(J=0) \rightarrow(J=1) \rightarrow(J=0)$ atomic radiative cascade. The excitation rate is more than ten times greater than that of Fry and Thompson. A great variety of tests were thus performed. In tests analogous to previous ones, we have attained a statistical accuracy never heretofore achieved. Data are directly compared to the QM predictions for the full $360^{\circ}$ range of relative orientations of the polarizers. Moreover, we have carried out a more general test of Bell's inequalities that does not require the assumption of rotational invariance.

The experiment was performed for various distances between the source and the polarizers. For large separations, our results are able to rule out various hypotheses ${ }^{12,13}$ according to which a nonfactorizing pure state for two particles (such as a singlet state) evolves towards a mixture of factorizing states when the two particles separate. Accordingly, such a localization process then occurs over distances of the order of the coherence length of the wave packets associated with the emitted photons. Such a hypothesis has already been tested previously using pairs of $\gamma$ rays, with conflicting results, ${ }^{9}$ but 
never in experiments employing visible photons. Following Freedman and Clauser, ${ }^{11}$ we used the $4 p^{2} S_{0}-4 s 4 p{ }^{1} P_{1}-4 s^{2}{ }^{1} S_{0}$ cascade in calcium (Fig. 1). This cascade yields two visible photons $\nu_{1}$ and $\nu_{2}$ correlated in polarization. Unlike their experiment, the calcium atoms are selectively pumped to the upper level of the cascade from the ground state by two-photon absorption (Fig. 1).

The experimental arrangement is shown in Fig.

2. An atomic beam of calcium is irradiated at $90^{\circ}$ by two laser beams focused at the interaction region, roughly a cylinder $1 \mathrm{~mm}$ long (atomic beam diameter) and $60 \mu \mathrm{m}$ diameter (laser beam waist diameter). A typical density at the interaction region is $3 \times 10^{10}$ atoms $/ \mathrm{cm}^{3}$, low enough to prevent any trapping of the resonant $422.7 \mathrm{~nm}$ light. ${ }^{14}$ The first laser beam $\left(\lambda_{K}=406.7 \mathrm{~nm}\right)$ is provided by a single-mode krypton ion laser, and the second laser is a cw single-mode Rhodamine $6 G$ dye laser tuned to resonance for the two-photon process $\left(\lambda_{D}=581 \mathrm{~nm}\right)$. The two lasers have parallel polarizations. The narrow resonance of the excitation process ${ }^{15}$ (less than 50 $\mathrm{MHz}$ ) allows selective excitation of the even ${ }^{40} \mathrm{Ca}$ isotope of calcium preventing hyperfine structure from weakening the polarization correlations. ${ }^{16}$ A phase-lock loop using the fluorescence signal of the cascade controls the wavelength of the tunable laser to ensure the maximum fluorescence signal. A second feedback loop controls the krypton-laser power for a stable cascade emission rate. With $40 \mathrm{~mW}$ from each laser, a typical cascade rate is $4 \times 10^{7}$ per second. We thus have an efficient source, with a small size facilitating the design of the optics in the detection channels.

The fluorescent light is collected by large-aperture aspheric lenses $(f=40 \mathrm{~mm}$, diameter $=50$

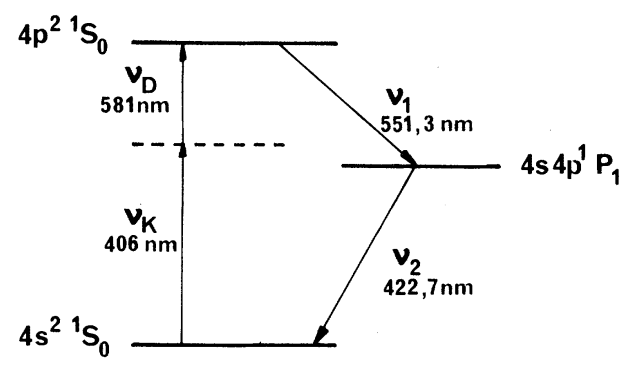

FIG. 1. Relevant levels of calcium. The atoms, selectively pumped to the upper level by the nonlinear absorption of $\nu_{K}$ and $\nu_{L}$, emits the photons $\nu_{1}$ and $\nu_{2}$ correlated in polarization. $\mathrm{mm}$ ), followed by a set of lenses for proper collimation. All the optical elements are antireflection coated. The stray light from the laser beams is reduced to a negligible level by various baffles. A colored-glass filter absorbs "the wrong wavelength" in front of each interference filter and prevents reflections of photons back towards the source and the other channel.

The polarizers I and II are "piles of plates" polarizers, consisting of ten optically flat glass plates inclined at nearly the Brewster's angle. Their efficiencies were directly measured in place in the experimental setup. The source was run and a linear sheet polarizer was inserted in front of each polarizer. The transmittances $\epsilon_{M}{ }^{i}$ and $\epsilon_{m}{ }^{i}$ of the polarizers ( $i=1$ or 2$)$ for light polarized parallel or perpendicular to the polar izer axis were then measured: $\epsilon_{M}{ }^{1}=0.971 \pm 0.005$, $\epsilon_{m}{ }^{1}=0.029 \pm 0.005, \epsilon_{M}{ }^{2}=0.968 \pm 0.005, \epsilon_{m}{ }^{2}=0.028$ \pm 0.005 . The photomultipliers have dark rates (less than 200 counts per second at $20^{\circ} \mathrm{C}$, no cooling was used) negligible compared with the typical singles counting rates (40000 and 120000 counts per second).

The coincidence-counting electronics includes a time-to-amplitude converter and a multichannel analyzer yielding the time-delay spectrum of the two-photon detections: a step at null delay followed by an exponential decrease with a time constant of 5 ns. Additionally, a standard coincidence circuit with a 19-ns coincidence window monitored the rate of coincidences around the null delay, while a second coincidence channel

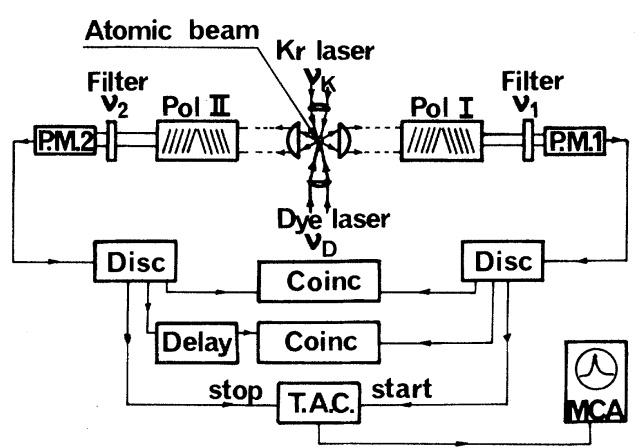

FIG. 2. Schematic diagram of apparatus and electronics. The laser beams are focused onto the atomic beam perpendicular to the figure. Feedback loops from the fluorescence signal control the krypton laser power and the dye-laser wavelength. The output of discriminators feed counters (not shown) and coincidence circuits. The multichannel analyzer (MCA) displays the time-delay spectrum. 
delayed in time by $100 \mathrm{~ns}$ monitored the accidental coincidences. The true coincidence rate determined by subtraction was found equal to the signal in the peak of the time-delay spectrum. Typical coincidence rates without polarizers are 240 coincidences per second in the null delay channel and 90 accidental coincidence per second; for a $100-$ s counting period we thus obtain 150 true coincidences per second with a standard deviation less than 2 coincidences per second.

The generalized Bell theorem ${ }^{2,3}$ yields the following inequalities:

$$
-1 \leqslant S=\left[R(\overrightarrow{\mathrm{a}}, \overrightarrow{\mathrm{b}})-R\left(\overrightarrow{\mathrm{a}}, \overrightarrow{\mathrm{b}}^{\prime}\right)+R\left(\overrightarrow{\mathrm{a}}^{\prime}, \overrightarrow{\mathrm{b}}\right)+R\left(\overrightarrow{\mathrm{a}}^{\prime}, \overrightarrow{\mathrm{b}}^{\prime}\right)-R_{1}\left(\overrightarrow{\mathrm{a}}^{\prime}\right)-R_{2}(\overrightarrow{\mathrm{b}})\right] / R_{0} \leqslant 0,
$$

where $R(\vec{a}, \vec{b})$ is the rate of coincidences with polarizer I in orientation $\vec{a}$ and polarizer II in orientation $\overrightarrow{\mathrm{b}}, R_{1}\left(\overrightarrow{\mathrm{a}}^{\prime}\right)$ is the coincidence rate with polarizer II removed and polarizer I in orientation $\overrightarrow{\mathrm{a}}^{\prime}$ [and similarly for $R_{2}(\overrightarrow{\mathrm{b}})$ ], and $R_{0}$ is the coincidence rate with the two polarizers removed. On the other hand, QM predicts ${ }^{2}$

$$
\begin{aligned}
& R(\varphi) / R_{0}=\frac{\hat{i}}{4}\left(\epsilon_{M}{ }^{1}+\epsilon_{m}{ }^{1}\right)\left(\epsilon_{M}{ }^{2}+\epsilon_{m}{ }^{2}\right)+\frac{1}{4}\left(\epsilon_{M}{ }^{1}-\epsilon_{m}{ }^{1}\right)\left(\epsilon_{M}{ }^{2}-\epsilon_{m}{ }^{2}\right) F(\theta) \cos 2 \varphi, \\
& R_{i} / R_{0}=\frac{1}{2}\left(\epsilon_{M}{ }^{i}+\epsilon_{m}{ }^{i}\right), \quad i=1 \text { or } 2 .
\end{aligned}
$$

Here, $\varphi=(\vec{a}, \vec{b})$ is the relative angle between the polarizer orientations, and $F(\theta)$ accounts for the finite solid angles subtended by the collecting lenses and takes the value $F=0.984$ for our ar rangement. A maximum violation of inequalities (1) is predicted by (2) for the sets of orientations shown in Fig. 3.

Assuming the rotational invariance of $R(\overrightarrow{\mathrm{a}}, \overrightarrow{\mathrm{b}})$, inequalities (1) contract to a form derived by Freedman,

$$
\delta=\left|R\left(22.5^{\circ}\right)-R\left(67.5^{\circ}\right)\right| / R_{0}-\frac{1}{4} \leqslant 0 .
$$

Figure 4 shows the results of a typical run. Here $R_{0}$ was measured for several periods of $100 \mathrm{~s}$; the dispersion of the results agrees with a Poisson law showing that the stability of the source is better than $0.5 \%$. The quantity $R(\varphi)$ was measured for each angle during a 100-s period. For supplementary checks, these measurements were repeated for angles between $0^{\circ}$ and $90^{\circ}$, and averaged. The standard deviation is estimated by statistical methods assuming a Poisson law. Our results clearly agree with the QM predictions (the solid curve) of Eq. (2).

The measurements at $22.5^{\circ}$ and $67.5^{\circ}$ involve
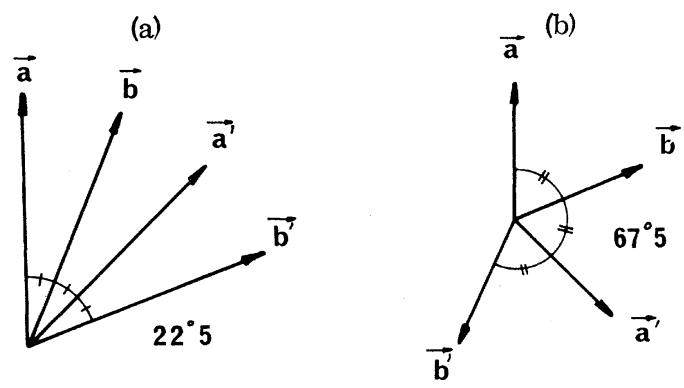

FIG. 3. Orientations leading to the maximum violations of generalized Bell inequalities. eight counting periods each, and yield

$$
\delta_{\text {exp }}=5.72 \times 10^{-2} \pm 0.43 \times 10^{-2},
$$

violating inequality (3) by more than 13 standard deviations, and in perfect agreement with $Q M$ prediction $\delta_{\mathrm{QM}}=5.8 \times 10^{-2} \pm 0.2 \times 10^{-2}$.

Although we never observed any deviation from rotational invariance, we have measured in a special run the quantities involved in $S$ [Eq. (1)] for one particular set of orientations as shown in Fig. 3(a). We found

$$
S_{\exp }=0.126 \pm 0.014 \text {, }
$$

violating inequality (1) by 9 standard deviations and in good agreement with $\mathrm{QM}$ prediction $S_{\mathrm{QM}}$ $=0.118 \pm 0.005$.

Last, moving each polarizer up to $6.5 \mathrm{~m}$ from

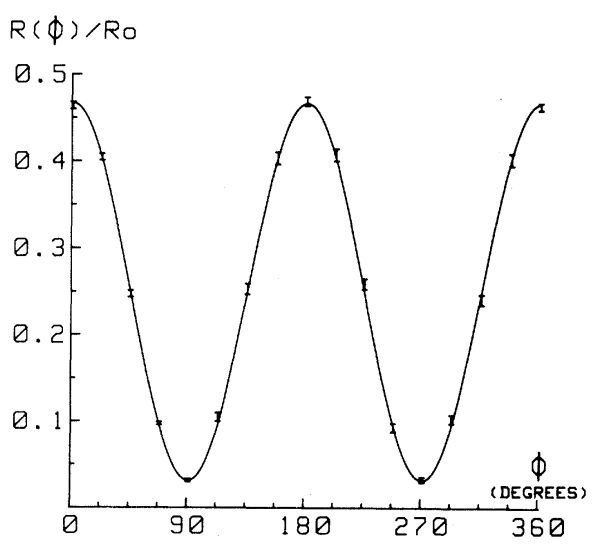

FIG. 4. Normalized coincidence rate as a function of the relative polarizer orientation. Indicated errors are \pm 1 standard deviation. The solid curve is not a fit to the data but the prediction of quantum mechanics. 
the source, i.e., to four coherence lengths of the wave packet associated with the lifetime of the intermediate state of the cascade ( $5 \mathrm{~ns}$ ), we observed no change in the results.

As a conclusion, our results, in excellent agreement with quantum mechanics predictions, are to a high statistical accuracy a strong evidence against the whole class of realistic local theories; furthermore, no effect of the distance between measurements on the correlations was observed.

The authors are very grateful to Professor C. Imbert, who sponsored this work. They acknowledge helpful discussions with Professor B. Cagnac for the design of the two-photon excitation of the cascade, and thank the many people who contributed to this work, especially Ing. A. Villing for the electronics, and our workshop personnel.

${ }^{1}$ J. S. Bell, Physics (N.Y.) 1, 195 (1965).

${ }^{2}$ J. F. Clauser, M. A. Horne, A. Shimony, and R. A. Holt, Phys. Rev. Lett. 23, 880 (1969).

${ }^{3}$ J. F. Clauser and M.A. Horne, Phys. Rev. D $\underline{10}$, 526 (1974).

${ }^{4} \mathrm{~J}$. S. Bell, in Foundations of Quantum Mechanics, edited by B. d'Espagnat (Academic, New York, 1972); B. d'Espagnat, Phys. Rev. D 11, 1424 (1975); P. Eberhard, Nuovo Cimento B38, 75 (1977). Other references can be found in J. F. Clauser and A. Shimony, Rep. Prog. Phys. 11 , 1881 (1978).
${ }^{5}$ Clauser and Shimony, Ref. 4. This paper is an exhaustive review of this question.

${ }^{6}$ D. J. Bohm, Quantum Theory (Prentice-Hall, Englewood Cliffs, N.J., 1951).

${ }^{7}$ A. Einstein, B. Podolsky, and N. Rosen, Phys. Rev. 47, 777 (1935).

${ }^{8}$ L. R. Kasday, J. D. Ullman, and C. S. Wu, Nuovo Cimento B25, 633 (1975); M. Bruno, M. d'Agostino, and C. Maroni, Nuovo Cimento B40, 142 (1977); K. Mesenheimer, thesis, University of Freiburg, 1979 (unpublished).

${ }^{9}$ G. Faraci, D. Gutkowski, S. Nottarigo, and A. R. Pennisi, Lett. Nuovo Cimento $\underline{9}, 607$ (1974); A. R. Wilson, J. Lowe, and D. K. Butt, J. Phys. G 2 , 613 (1976).

${ }^{10}$ M. Lamehi-Rachti and W. Mittig, Phys. Rev. D 14 , 2543 (1976).

${ }^{11}$ S. J. Freedman and J. F. Clauser, Phys. Rev. Lett. 28, 938 (1972); R. A. Holt and F. M. Pipkin, to be published; J. F. Clauser, Phys. Rev. Lett. 36,1223 (1976); E. S. Fry and R. C. Thompson, Phys. Rev. Lett. 37, 465 (1976)

${ }^{12}$ W. H. Furry, Phys. Rev. 49, 393 (1936).

${ }^{13} \mathrm{D}$. Bohm and Y. Aharonov, Phys. Rev. 108, 1070

(1957); L. de Broglie, C. R. Acad. Sci. 278B, 721

(1974); D. Bohm and B. J. Hiley, Nuovo Cimento B35, 137 (1976).

${ }^{14}$ Freedman and Clauser, Ref. 11, observed trapping at such densities but their atomic beam had greater transverse dimensions than ours.

${ }^{15} \mathrm{~A}$. A spect, C. Imbert, and G. Roger, Opt. Commun. 34, $46(1980)$.

${ }^{16}$ E. S. Fry, Phys. Rev. A $\underline{8}, 1219$ (1973).

\title{
Gravitational and Inertial Effects in Quantum Fluids
}

\author{
J. Anandan \\ Department of Mathematics, University of California, Berkeley, California 94720
}

(Received 27 March 1981)

A general theory of the interaction of superfluid helium with a gravitational field is developed within the framework of general relativity by using a covariant generalization of the Gross-Pitaevskii equation. The general relativistic Sagnac effect for the superfluid Josephson interferometer is obtained in the stationary case. The influence of a plane-polarized gravitational wave on a recently proposed superfluid gravitational antenna as well as a new antenna is determined.

PACS numbers: 04.80.+z, 03.65.-w, 67.40.Bz

The possibility of using quantum interference effects in superfluid helium to detect gravitational and inertial perturbations, including gravitational radiation, has been proposed recently. ${ }^{1,2}$ This raises the exciting possibility of testing general relativity in the laboratory at the quantum mechanical level for the first time. In this Letter, I shall therefore develop the general theory of the influence of gravity and inertia on super- fluid helium from a general relativistic point of view. I shall then obtain the phase shift that gives rise to a Josephson current for the specific cases of the stationary Sagnac effect and the interaction of a plane gravitational wave with two simple superfluid gravitational antennas.

The proposed experiments may also be important for the study of superfluid helium because of the absence of a satisfactory microscopic theory 